\title{
DNA Methylation in Neurodegenerative Disorders
}

\author{
Zihui Xu • Xuekun Li
}

Published online: 22 September 2012

(C) Springer Science+Business Media, LLC 2012

\begin{abstract}
Epigenetic mechanisms are essential in a host of biological processes and many different human diseases. Epigenetic modifications include DNA methylation, histone modification, non-coding RNA, and nucleosome positioning. In neuronal systems, extensive studies have revealed important regulatory roles of DNA methylation in brain function, from the embryonic stage through the process of aging. Here we review recent evidence that DNA methylation is involved in aging, learning and memory, and neurodegenerative diseases, including Alzheimer's disease, Parkinson's disease, amyotrophic lateral sclerosis, and CGG repeat-induced neurodegenerative disorders. We also explore the dynamics of DNA methylation.
\end{abstract}

Keywords DNA methylation · Neurodegeneration · Cognitive function · Aging · Alzheimer's disease . Parkinson's disease · Amyotrophic lateral sclerosis · DNA demethylation

\section{Introduction}

Epigenetics refers to modifications that lead to heritable phenotypes, without affecting the DNA sequence itself [1]. Epigenetic modifications include DNA methylation, histone modifications, the action of small RNAs, and nucleosome positioning $[2,3,4 \bullet \bullet$. In mammals, DNA methylation is for

Z. Xu $\cdot$ X. Li $(\bowtie)$

Department of Human Genetics,

Emory University School of Medicine,

Atlanta, GA, USA

e-mail: xuekun.li@emory.edu

\section{Z. Xu}

Division of Histology and Embryology, Department of Anatomy,

Tongji Medical College,

Huazhong University of Science and Technology,

Wuhan, People's Republic of China the most part cytosine methylation [5-methylcytosine $(5 \mathrm{mC})]$, and is found predominantly within $\mathrm{CpG}$ dinucleotides. The DNA methylation machinery in mammals consists of two components: the DNA methyltransferases (DNMTs), which establish and maintain DNA methylation patterns, and the methyl-CpG binding proteins (MBDs), which are involved in "reading" methylation marks [5]. DNA methylation is catalyzed by a family of DNA methyltransferases (DNMTs) that transfer a methyl group from Sadenyl methionine (SAM) to the fifth carbon of a cytosine residue to form $5 \mathrm{mC}$. Five members of the DNMT family have been reported in mammals: DNMT1, DNMT2, DNMT3a, DNMT3b, and DNMT3L [4••]. DNMT3a and DNMT3b can establish a new methylation pattern in unmodified DNA and are thus known as de novo DNMTs. The de novo DNMTs are responsible for establishing the normal pattern of methylation during embryonic development [6]. DNMT3L, despite being catalytically inactive, is required for establishing maternal genomic imprinting [7]. It acts as a general stimulatory factor for DNMT3a and DNMT3b, and interacts and colocalizes with them in the nucleus [8, 9]. DNMT1, the maintenance enzyme of DNA methylation, is the most abundant DNMT and is transcribed primarily during the S-phase of the cell cycle.

In mammalian neuronal systems, DNA methylation has been implicated in the regulation of neural stem cell fate determination, brain development, cognitive function, neurodevelopmental disorders, and neurodegenerative diseases [10-15]. The absence of DNMT1 has a lethal effect in mice, and DNMT3a can regulate neurogenic capability by regulating the neuronal transcriptome. DNA methylation is also associated with the formation of memory. In the context of disease, DNA methylation is involved in neuronal cell death and ischemia-induced injury. Recent studies have shown that DNA methylation plays important roles in a range of human diseases, including neurodegenerative disorders. Here, we will provide an overview of recent findings that 
shed light on the function of DNA methylation in multiple neurodegenerative diseases. We will also discuss the dynamics of DNA methylation.

\section{DNA Methylation and Aging}

Aging is defined as a loss of phenotypic plasticity over time. At a cellular level, aging is characterized by oxidative stress, disturbed calcium homeostasis, chromosomal instability, impaired DNA repair, and the accumulation of nuclear and mitochondrial DNA damage [16]. Combined or individually, these factors can lead to neuronal aging, neurodegeneration, and cell death in the central nervous system. DNA methylation is known to play important roles in fundamental cellular processes such as differentiation, proliferation, and senescence by modulating both local and genome-wide gene transcription [5]. The aging cell is found to undergo a DNA methylation drift, with generally an overall decrease in total genomic $5 \mathrm{mC}$ content in the brain [17]. Studies in cultured aging human fibroblasts have revealed that DNMT1 expression and activity are gradually reduced with increased population doublings [18].

It is now well established that aberrant DNA methylation processes are often a causal factor in several major neurological disorders [5]. A number of recent studies identified abnormal DNA methylation events in neuronal cells associated with aging and age-related neurodegenerative disorders, such as Alzheimer's disease [19-21]. Dnmt1 haploinsufficiency (Dnmt1+/-) can cause premature aging effects and poor cognitive function without affecting survival or mortality. Further, DNA methylation decreases with normal aging and is also lower in Dnmt1+/-mouse brain tissues than in Dnmt1+/+ mouse brain tissues [22]. A recent study in elderly subjects revealed a gradual age-dependent loss of genomic DNA methylation within the same individual over an eight-year span [23]. The DNA methylation landscape of $\mathrm{CD} 4+\mathrm{T}$ cells from newborn and centenarian individuals showed that DNA obtained from a 103year-old donor was more unmethylated overall than DNA from the same cell type obtained from a neonate [24•]. Furthermore, the centenarian sample showed a lower correlation in terms of the methylation status of neighboring CpGs versus the newborn sample, which was more homogenously methylated in nearby located CpGs. The hypomethylated CpGs observed in the centenarian DNA covered all genomic compartments, such as promoters, exonic, intronic, and intergenic regions [24•]. These longitudinal studies provide important in vivo evidence that loss of DNA methylation indeed occurs with age under normal physiological conditions in humans.

\section{DNA Methylation and Learning and Memory}

Synaptic connectivity among neurons serves as the physical basis for memory formation, which often involves gene products (mRNA or protein) from a vast number of neural activity-related genes. DNMT activity is required for associative memory formation and induction of long-term potentiation (LTP) [25, 26]. Infusion of the DNMT inhibitors 5-aza-deoxycytidine (5-AZA) or zebularine into the hippocampus immediately after contextual fear conditioning reverses the methylation and downregulation of PP1, a memory suppressor gene, impairing the formation of fear memory [25]. Interestingly, methylation levels in the hippocampus return to baseline in 24 hours, although the fear memory is maintained and is still dependent on the hippocampus at that time point [25]. This indicates that, in the hippocampus, DNA methylation might not be a mechanism of contextual fear memory maintenance, but a regulatory mechanism of transient gene expression. Furthermore, cocaine-induced conditioned place preference memory was recently found to require DNA methylation in the hippocampus $[27 \bullet \bullet]$.

To assess the role of DNA methylation in the mature mouse brain, Dnmt1 was deleted specifically in the precursors of postnatal excitatory neurons in the dorsal forebrain. The mutant mice showed abnormal development of the somatosensory barrel cortex and impaired thalamocortical long-term potentiation [28]. When Dnmt1 is deleted under the control of the calmodulin-kinase IIa (CaMKIIa) promoter [29], neither the DNA methylation level of endogenous retroviral repeats nor neuronal survival is affected. Interestingly, double knockout of Dnmt1 and Dnmt3a mediated by the CaMKIIa-cre system results in smaller cell sizes, impaired hippocampal LTP, enhanced LTD, and deficits in spatial and contextual fear memory formation, whereas single knockout lines of each gene display no such abnormalities [30]. In particular, Stat1, which is involved in neural plasticity and the interferon pathway, is upregulated at the mRNA level and decreases at the methylation level in neuronal cells. These results indicate that DNA methylation is important for synaptic plasticity and learning, probably by affecting the expression of plasticityrelated genes.

Although some genes, including brain-derived neurotrophic factor (BDNF), are demethylated, with a corresponding increase in mRNA after learning, no study has established whether DNA demethylation is necessary for memory consolidation. This is because the demethylation mechanism remains unclear. One recent interesting report described how the activity-induced gene Gadd $45 \mathrm{~b}$ is required for activity-dependent upregulation of BDNF [31]. Further studies of the roles of DNA demethylation in the learning and memory paradigm would be of great interest. 
A recent report has demonstrated that DNA methylation is required for the maintenance of memory [32•]. Contextual fear memory formation and its initial maintenance depend on the hippocampus, but it is generally believed that memory undergoes systems consolidation over approximately three weeks, so that the remote memory becomes dependent on the prefrontal cortex, including the anterior cingulate cortex (ACC), and independent of the hippocampus [33, 34]. To test whether memory maintenance requires DNA methylation, Miller et al. looked at the ACC region, rather than the hippocampus [32•]. After contextual fear conditioning, hypermethylation of the calcineurin $(\mathrm{CaN})$ gene was maintained for at least 30 days; a correlative decrease of calcineurin mRNA and protein also persisted for at least a month. When a DNMT inhibitor was injected into the ACC 29 days after training, DNA methylation on CaN decreased, and 30-day memory was impaired. These findings suggest that the DNA methylation and demethylation processes are ongoing in the ACC region and that this dynamic balance is required for memory maintenance. Similarly, retrieval of conditioned place preference memory was recently found to depend on DNA methylation in the prelimbic cortex $[27 \bullet \bullet]$.

The question is how the modification of DNA in the nucleus, which has a cell-wide effect, could be involved in maintaining a specific memory. A neuron has thousands of synapses connecting to a number of other neurons, and there is a high likelihood that it participates in multiple memories through different synapses [15]. It seems quite certain that modification of DNA in the nucleus itself cannot differentially affect each synapse without synapsespecific changes. Therefore, DNA methylation in itself may be insufficient for storing memory, and the exact mechanisms are still open for exploration.

\section{DNA Methylation and Alzheimer's Disease}

Alzheimer's disease (AD) is a slow, progressive dementia affecting primarily the cortex and hippocampus, characterized by the deposit of abnormal filaments of hyperphosphorylated Tau protein in neuronal cytoplasm, known as neurofibrillary tangles, accompanied by extracellular aggregates of large amyloid $\beta$ protein $(A \beta)$, known as senile plaques [35]. DNA methylation has been proposed as a mechanism that can lead to aberrant changes in gene expression during the progression of $\mathrm{AD}$. In several $\mathrm{AD}$ brain studies, the loss of DNA methylation has important consequences. Furthermore, elevated levels of Sadenosylhomocysteine, a potential inhibitor of methyltransferases, have been detected in AD brains, and are inversely correlated with patient cognition, implicating epigenetic changes during the progression of AD [36]. DNA hypomethylation in promoter $\mathrm{CpGs}$ of $\mathrm{AD}$-associated genes, such as APP, presenilin 1(PS1), and $\beta$-site APPcleaving enzyme1 (BACE1), is also seen in AD brains [37], and results in abnormal upregulation of these genes, leading to excess accumulation of $A \beta$. In addition, an increased level of methylation at specific loci in the promoter region of certain genes, such as apolipoprotein E and methylenetetrahydrofolate reductase, is also found in the cortex of AD brains versus controls [17]. This suggests that the simultaneous occurrence of hyper/hypomethylation may contribute to the pathogenesis of AD.

As mentioned previously, aging is the major known risk factor for $\mathrm{AD}$, and is associated with multiple epigenetic alterations [38]. Aging-related DNA methylation may represent one of the mechanisms by which environmental and dietary factors can promote AD [39]. Some cytosines in the promoter region of the APP gene are frequently methylated in patients who are under 70 years old, and these methylated cytosines are significantly demethylated in cases over the age of 70. These age-related modifications to DNA methylation alter APP expression and consequently can affect the progressive $A \beta$ deposition with aging in the brain [40].

Age-related epigenetic modifications in $\mathrm{AD}$ were further explored in two other studies. The first study presented straightforward evidence of epigenetic involvement in AD pathogenesis, by showing an age-specific epigenetic drift in late-onset $\mathrm{AD}$. PSEN1 and APOE, which participate in $\mathrm{A} \beta$ processing, and MTHFR and DNMT1, which are responsible for methylation homeostasis, demonstrated a significant interindividual epigenetic variability in the brain and lymphocytes of these patients, which could contribute to the predisposition to late-onset $\mathrm{AD}$ [17]. In the second study, longevity-related genes were investigated with respect to promoter methylation in peripheral blood in relation to gender, age, and AD status. Only one of the genes, HTERT, was shown to be hypermethylated in AD versus aged normal people. This gene, which is transcribed into the mRNA component of telomerase, and unlike the regular effect of methylation on gene expression, is activated by this epigenetic modification. Consequently, these results indicated higher telomerase activity, likely due to telomere and immune dysfunctions involved in AD pathogenesis [20].

\section{DNA Methylation and Parkinson's Disease}

Parkinson's disease (PD) is the second most common neurodegenerative disorder after $\mathrm{AD}$, with an average age of onset of 60 years, and according to the Parkinson's Disease Foundation, it affects about 1 million people in the United States and more than 4 million people worldwide [41]. Pathologically, PD is characterized by the loss of dopaminergic neurons in the substantia nigra (SN) pars compacta, 
and patients frequently have Lewy bodies, eosinophilic intracellular inclusions composed of amyloid-like fibers, and $\alpha$-synuclein (SNCA) [42].

Thus far, there have been fewer epigenetic studies performed in PD versus AD subjects; however, there is some indication of impaired one-carbon metabolism in PD, as well as altered DNA methylation potential $[43,44]$. Epigenetic analyses of PD brains reveal that the SNCA gene could be subjected to epigenetic regulation $[45 \cdot, 46]$. Several lines of evidence, including the identification of families with SNCA locus duplication and triplication and the association of both promoter and 3' UTR polymorphisms with sporadic forms, point to a gene-dosage effect for SNCA in PD pathogenesis [47]. The analysis of SNCA alleles in a PD patient heterozygous for the A53T mutation, the first mutation to be implicated in PD pathogenesis, revealed that SNCA showed monoallelic expression in this patient, with epigenetic silencing of the mutated allele due to histone modifications, but not DNA methylation, and upregulation of the wild-type allele resulting in higher mRNA levels than in matched control subjects [48]. Others have found that the methylation of human SNCA intron 1 decreases gene expression, whereas inhibition of DNA methylation activates SNCA expression. They also show that DNA methylation of SNCA intron 1 is reduced in several brain regions of sporadic PD patients, including in the substantia nigra, putamen, and cortex, pointing towards an epigenetic regulation of SNCA expression in PD [45 ${ }^{\circ}$. Another research group identified an SNCA CpG island in which the methylation status is altered, along with increased SNCA expression. Postmortem brain analysis revealed regional nonspecific methylation differences in this $\mathrm{CpG}$ region in the anterior cingulate and putamen among controls and PD patients; however, in the substantia nigra of PD individuals, the methylation of this region was significantly decreased [46]. Both findings are consistent with previous reports indicating increased SNCA mRNA levels in PD substantia nigra tissue. A recent paper suggested that $\alpha$-synuclein sequesters DNMT1 from the nucleus [49•]. These researchers observed a reduction of nuclear DNMT1 levels in human postmortem brain samples from PD patients and from patients with dementia with Lewy bodies (DLBs), as well as in the brains of $\alpha$-synuclein transgenic mice models. Furthermore, sequestration of DNMT1 in the cytoplasm resulted in global DNA hypomethylation in human and mouse brains, involving $\mathrm{CpG}$ islands upstream of SNCA and other genes. The nuclear DNMT1 levels were partially rescued by overexpression of DNMT1 in neuronal cell cultures and in $\alpha$-synuclein transgenic mouse brains. Therefore, the authors suggested that the association of DNMT1 and $\alpha$-synuclein might mediate aberrant subcellular localization of DNMT1, resulting in epigenetic modifications in the brain [49•].

\section{Amyotrophic Lateral Sclerosis}

Amyotrophic Lateral Sclerosis (ALS) is a fatal, adult-onset disease, and most cases are sporadic, with no known inherited component. Although epidemiological studies implicate some environmental and acquired factors in its pathogenesis, previous genetic studies have been unable to identify bona fide genes responsible for the molecular mechanisms underlying sporadic ALS. DNA methylation in sporadic ALS has been examined in two candidate genes, SOD1 and VEGF [50]; in members of the metallothionein gene family [51]; and in the astroglial EAAT2 gene promoter [52]. None of these studies detected differences in methylation patterns in sporadic ALS cases compared with control cases. Another study using genome-wide analysis of brain DNA methylation in sporadic ALS, which involved chromatin immunoprecipitation followed by $\mathrm{CpG}$ microarray hybridization, revealed significant hypermethylation of genes involved in calcium dynamics, oxidative stress, and synapses [53]. Most recently, aberrant regulation of DNA methylation has been linked to the pathobiology of ALS [54••]. In human sporadic ALS, DNMT1 and DNMT3a proteins are upregulated in motor cortex and spinal cord motor neurons. Moreover, DNMT3a protein levels are increased in mitochondrial fractions, which have a particularly prominent pro-apoptotic function in motor neurons.

\section{DNA Methylation and Trinucleotide Repeat Disorders}

The expansion of trinucleotide repeats (TNRs) during gametogenesis leads to mutation or silencing of associated genes, with pathological consequences [55]. Although the causes of TNR instability are largely mysterious, DNA polymerase slippage, DNA repair mechanisms, transcription, and nucleosome positioning within the repeat are all candidates [56].

CAG repeat expansion is the underlying cause of at least 12 neurodegenerative diseases [57]. We do not know what effect DNA methylation might have on CAG repeats, which themselves are not targets for DNA methylation since they contain no CG dinucleotides. Nevertheless, three observations point to a connection between DNA methylation and CAG repeat stability. First, in bacteria, CAG repeats in plasmids methylated at $\mathrm{CpG}$ sites were mildly stabilized relative to repeats in unmethylated plasmids [58]. Second, treatment of mammalian cells with 5-aza-2'-deoxycytosine (5-aza-CdR), which leads to passive depletion of DNA methylation and destruction of Dnmt1 [59], or with hydralazine, which induces demethylation by inhibiting expression of Dnmt1 [59], both dramatically increased CAG repeat instability [60]. Finally, another study provided a mechanistic link between DNA methylation and repeat stability. 
Vincent Dion et al. found that Dnmt1 deficiency in mice promoted intergenerational expansion of CAG repeats at the murine spinocerebellar ataxia type 1 (SCA1) locus. Importantly, Dnmt1+/- SCA1 mice, unlike their Dnmt1+/+ SCA1 counterparts, closely reproduced the intergenerational instability patterns. In addition, this group also found aberrant DNA and histone methylation at sites within the $\mathrm{CpG}$ islands that abut the expanded repeat tract in Dnmt1deficient mice [61].

Expansion of methylatable CGG repeats occurs at several sites that are either known or likely to be involved in human disease, and increased DNA methylation is thought to play a role in pathogenesis. The CGG repetitive element as well as an upstream $\mathrm{CpG}$ island in the FMR1 promoter is abnormally hypermethylated in most individuals affected with fragile X syndrome. Initially, this methylation was thought to be the primary mediator of epigenetic silencing, with secondary recruitment of histone deacetylases and methyltransferases driving formation of a heterochromatin region over the FMR1 locus. Indeed, this DNA methylation pattern is found to be associated with histone deacetylation and heterochromatin formation across the FMR1 gene in differentiated cells $[62,63]$.

A connection between DNA methylation and repeat instability has also been noted for the expanded CGG repeats in the 5' end of the FMR1 gene. In somatic tissues, methylated CGG repeat tracts at the FMR1 locus are found to be much more stable than unmethylated ones of comparable length [64], and when vectors carrying CGG repeats were introduced into primate cells, or into bacteria, methylated repeats proved to be more stable than unmethylated ones [58].

\section{DNA Methylation and Demethylation}

For decades, DNA methylation was believed to be a stable and irreversible modification that could not be further modified. Although Wilson and Jones implicated DNA methylation dynamics during aging in 1983, the demethylation enzymes had been chased since then. In 2009, the discovery of 5-hydroxymethylcytosine $(5 \mathrm{hmC})$ in mammals suggested DNA demethylation in the brain $[65,66]$. Oxidation of $5 \mathrm{mC}$ to $5 \mathrm{hmC}$ by ten-eleven translocation (Tet) enzymes is proposed as an intermediate step in active DNA demethylation. Tet proteins can further oxidize $5 \mathrm{hmC}$ to 5 -formylcytosine (5fC) and 5-carboxylcytosine $(5 \mathrm{caC})$, and $5 \mathrm{caC}$ can be excised further by a thymine-DNA glycosylase (TDG)-mediated base excision repair mechanism [67••, 68]. In mouse preimplant zygotes, an increase in $5 \mathrm{hmC}$ is associated with a decrease in $5 \mathrm{mC}$, suggesting that $5 \mathrm{mC}$ is actively converted to $5 \mathrm{hmC}$ by Tet3, which is the only isoform of Tet protein expressed in zygotes [69]. Moreover, the $5 \mathrm{hmC}$ mark associated with the paternal genome in zygotes is gradually lost in a DNA replication-dependent manner during preimplantation zygote development [70]. It remains to be seen whether such active and passive mechanisms of $5 \mathrm{hmC}$ mediated DNA demethylation could have a specific function in the brain.

\section{Conclusion}

DNA methylation plays important roles in common human neurodegenerative diseases, which means modulating DNA methylation could be a potential therapeutic strategy for such diseases. Profiling DNA methylation at a genomewide level could help us understand the basis of neurodegenerative disorders, including AD and PD. Furthermore, the integration of genome-wide DNA methylation maps with other epigenetic profiling should give us more insight into the function of DNA methylation in human diseases.

Acknowledgments The authors would like to thank Dr. Peng Jin and C.Strauss for critical reading of the manuscript. X. Li is supported by the Autism Speaks foundation (\# 7660).

Disclosure No potential conflicts of interest relevant to this article were reported.

\section{References}

Papers of particular interest, published recently, have been highlighted as:

- Of importance

-• Of major importance

1. Wu C, Morris JR. Genes, genetics, and epigenetics: a correspondence. Science. 2001;293(5532):1103-5.

2. Zaratiegui M, Irvine DV, Martienssen RA. Noncoding RNAs and gene silencing. Cell. 2007;128(1):763-76.

3. Bai L, Morozov AV. Gene regulation by nucleosome positioning. Trends Genet. 2010;26(11):476-83.

4. .- Portela A, Esteller M. Epigenetic modifications and human disease. Nat Biotechnol. 2010;28(10):1057-68. This paper reviewed epigenetic modifications and their machineries in neurodegenerative and neurological diseases.

5. Robertson KD. DNA methylation and human disease. Nat Rev Genet. 2005;6(8):597-610.

6. Esteller, M. Epigenetic gene silencing in cancer: the DNA hypermethylome. Hum Mol Genet, 2007. 16 Spec No 1:R50-9.

7. Bourc'his D, et al. Dnmt3L and the establishment of maternal genomic imprints. Science. 2001;294(5551):2536-9.

8. Chen ZX, et al. Physical and functional interactions between the human DNMT3L protein and members of the de novo methyltransferase family. J Cell Biochem. 2005;95(5):902-17.

9. Purdy MM, Holz-Schietinger C, Reich NO. Identification of a second DNA binding site in human DNA methyltransferase 3A by substrate inhibition and domain deletion. Arch Biochem Biophys. 2010;498(1):13-22. 
10. Hirabayashi Y, Gotoh Y. Epigenetic control of neural precursor cell fate during development. Nat Rev Neurosci. 2010;11(6):377-88.

11. Dulac C. Brain function and chromatin plasticity. Nature. 2010;465 (7299):728-35.

12. Day JJ, Sweatt JD. Epigenetic mechanisms in cognition. Neuron. 2011;70(5):813-29.

13. Ma DK, et al. Epigenetic choreographers of neurogenesis in the adult mammalian brain. Nat Neurosci. 2010;13(11):1338-44.

14. Urdinguio RG, Sanchez-Mut JV, Esteller M. Epigenetic mechanisms in neurological diseases: genes, syndromes, and therapies. Lancet Neurol. 2009;8(11):1056-72.

15. Day JJ, Sweatt JD. DNA methylation and memory formation. Nat Neurosci. 2010;13(11):1319-23.

16. Chouliaras L, et al. Epigenetic regulation in the pathophysiology of Alzheimer's disease. Prog Neurobiol. 2010;90(1):498-510.

17. Wang SC, Oelze B, Schumacher A. Age-specific epigenetic drift in late-onset Alzheimer's disease. PLoS One. 2008;3(7):e2698.

18. Casillas Jr MA, et al. Transcriptional control of the DNA methyltransferases is altered in aging and neoplastically-transformed human fibroblasts. Mol Cell Biochem. 2003;252(1-2):33-43.

19. Siegmund KD, et al. DNA methylation in the human cerebral cortex is dynamically regulated throughout the life span and involves differentiated neurons. PLoS One. 2007;2(1):e895.

20. Silva PN, et al. Promoter methylation analysis of SIRT3, SMARCA5, HTERT and CDH1 genes in aging and Alzheimer's disease. J Alzheimers Dis. 2008;13(2):173-6.

21. Mastroeni D, et al. Epigenetic changes in Alzheimer's disease: decrements in DNA methylation. Neurobiol Aging. 2010;31 (12):2025-37.

22. Liu L, et al. Insufficient DNA methylation affects healthy aging and promotes age-related health problems. Clin Epigenetics. 2011;2(2):349-60.

23. Bollati V, et al. Decline in genomic DNA methylation through aging in a cohort of elderly subjects. Mech Ageing Dev. 2009;130(1):234-9.

24. - Heyn H, et al. Distinct DNA methylomes of newborns and centenarians. Proc Natl Acad Sci U S A. 2012;109(26):10522-7. This paper performed whole-genome bisulfite sequencing (WGBS) of newborn and centenarian genomes using a 450,000 CpG-site DNA methylation microarray that reinforced the observation of more hypomethylated DNA sequences in the advanced age group.

25. Miller CA, Sweatt JD. Covalent modification of DNA regulates memory formation. Neuron. 2007;53(6):857-69.

26. Levenson JM, et al. Evidence that DNA (cytosine-5) methyltransferase regulates synaptic plasticity in the hippocampus. J Biol Chem. 2006;281(23):15763-73.

27. • Han J, et al. Effect of 5-aza-2-deoxycytidine microinjecting into hippocampus and prelimbic cortex on acquisition and retrieval of cocaine-induced place preference in C57BL/6 mice. Eur J Pharmacol. 2010;642(1-3):93-8. This paper reported the injection of the DNA methyltransferases (DNMTS) inhibitor 5-aza-2-deoxycytidine (5-aza) into the hippocampus CA1 area and prelimbic cortex during the stages of acquisition and expression of cocaine-induced place preference in C57BL/6 mice. Results showed that in CA1, DNA methylation inhibitors could restrain acquisition but had no impact on expression of the cocaine-induced conditioned place preference (CPP). On the contrary, in prelimbic cortex, 5-aza had no effect on acquisition but blocked expression.

28. Golshani P, et al. Conditional Dnmt1 deletion in dorsal forebrain disrupts development of somatosensory barrel cortex and thalamocortical long-term potentiation. Thalamus Relat Syst. 2005;3(3):227-33.

29. Fan G, et al. DNA hypomethylation perturbs the function and survival of CNS neurons in postnatal animals. J Neurosci. 2001;21 (3):788-97.

30. Feng J, et al. Dnmt1 and Dnmt3a maintain DNA methylation and regulate synaptic function in adult forebrain neurons. Nat Neurosci. 2010;13(1):423-30.
31. Ma DK, et al. Neuronal activity-induced Gadd45b promotes epigenetic DNA demethylation and adult neurogenesis. Science. 2009;323(5917):1074-7.

32. - Miller CA, et al. Cortical DNA methylation maintains remote memory. Nat Neurosci. 2010;13(6):664-6. This paper reported that persistent, gene-specific cortical hypermethylation was induced in rats by a single, hippocampus-dependent associative learning experience, and pharmacologic inhibition of methylation 1 month after learning disrupted remote memory.

33. Frankland PW, et al. The involvement of the anterior cingulate cortex in remote contextual fear memory. Science. 2004;304 (5672):881-3.

34. Frankland PW, Bontempi B. The organization of recent and remote memories. Nat Rev Neurosci. 2005;6(2):119-30.

35. Blennow K, de Leon MJ, Zetterberg H. Alzheimer's disease. Lancet. 2006;368(9533):387-403.

36. Kennedy BP, et al. Elevated S-adenosylhomocysteine in Alzheimer brain: influence on methyltransferases and cognitive function. J Neural Transm. 2004;111(1):547-67.

37. Fuso A, et al. S-adenosylmethionine/homocysteine cycle alterations modify DNA methylation status with consequent deregulation of PS1 and BACE and beta-amyloid production. Mol Cell Neurosci. 2005;28(1):195-204.

38. Ferri CP, et al. Global prevalence of dementia: a Delphi consensus study. Lancet. 2005;366(9503):2112-7.

39. Fuso A, et al. Changes in Presenilin 1 gene methylation pattern in diet-induced B vitamin deficiency. Neurobiol Aging. 2011;32 (2):187-99.

40. Tohgi $\mathrm{H}$, et al. Reduction with age in methylcytosine in the promoter region -224 approximately -101 of the amyloid precursor protein gene in autopsy human cortex. Brain Res Mol Brain Res. 1999;70(2):288-92.

41. de Lau LM, Breteler MM. Epidemiology of Parkinson's disease. Lancet Neurol. 2006;5(6):525-35.

42. Weintraub D, Comella CL, Horn S. Parkinson's disease-Part 1: Pathophysiology, symptoms, burden, diagnosis, and assessment. Am J Manag Care. 2008;14(2 Suppl):S40-8.

43. Obeid R, et al. Methylation status and neurodegenerative markers in Parkinson disease. Clin Chem. 2009;55(10):1852-60.

44. Herrmann W, Obeid R. Biomarkers of folate and vitamin B(12) status in cerebrospinal fluid. Clin Chem Lab Med. 2007;45 (12):1614-20.

45. - Jowaed A, et al. Methylation regulates alpha-synuclein expression and is decreased in Parkinson's disease patients' brains. J Neurosci. 2010;30(18):6355-9. This paper showed that methylation of human SNCA intron 1 decreased gene expression, while inhibition of DNA methylation activated SNCA expression. Methylation of SNCA intron 1 was reduced in DNA from sporadic PD patients'substantia nigra, putamen, and cortex, pointing toward an as-yet unappreciated epigenetic regulation of SNCA expression in $P D$.

46. Matsumoto L, et al. $\mathrm{CpG}$ demethylation enhances alpha-synuclein expression and affects the pathogenesis of Parkinson's disease. PLoS One. 2010;5(11):e15522.

47. Chartier-Harlin MC, et al. Alpha-synuclein locus duplication as a cause of familial Parkinson's disease. Lancet. 2004;364 (9440):1167-9.

48. Voutsinas GE, et al. Allelic imbalance of expression and epigenetic regulation within the alpha-synuclein wild-type and p.Ala53Thr alleles in Parkinson disease. Hum Mutat. 2010;31(6):685-91.

49. - Desplats P, et al. Alpha-synuclein sequesters Dnmt1 from the nucleus: a novel mechanism for epigenetic alterations in Lewy body diseases. J Biol Chem. 2011;286(11):9031-7. This paper presented evidence of a reduction in nuclear Dnmt1 levels in human postmortem brain samples from $P D$ and Lewy bodies (DLB) patients, as well as in the brains of $\alpha$-synuclein transgenic 
mice models. Furthermore, sequestration of Dnmt1 in the cytoplasm results in global DNA hypomethylation in human and mouse brains, involving CPG islands upstream of the SNCA, SEPW1, and PRKAR2A genes.

50. Oates N, Pamphlett R. An epigenetic analysis of SOD1 and VEGF in ALS. Amyotroph Lateral Scler. 2007;8(2):83-6.

51. Morahan JM, et al. Are metallothionein genes silenced in ALS? Toxicol Lett. 2007;168(1):83-7.

52. Yang Y, et al. Epigenetic regulation of neuron-dependent induction of astroglial synaptic protein GLT1. Glia. 2010;58(3):277-86.

53. Morahan JM, et al. A genome-wide analysis of brain DNA methylation identifies new candidate genes for sporadic amyotrophic lateral sclerosis. Amyotroph Lateral Scler. 2009;10(5-6):418-29.

54. •• Chestnut BA, et al. Epigenetic regulation of motor neuron cell death through DNA methylation. J Neurosci. 2011;31(46):1661936. This paper showed enforced expression of Dnmt3a induced degeneration of cultured NSC34 cells. Truncation mutation of the Dnmt3a catalytic domain and Dnmt3a RNAi blocked apoptosis of cultured neurons. Inhibition of Dnmt catalytic activity with RG108 and procainamide protected cultured neurons from excessive DNA methylation and apoptosis. Dnmt1 and Dnmt3a are expressed in motor neurons of adult mouse spinal cord, and, during their apoptosis induced by sciatic nerve avulsion, nuclear and cytoplasmic 5-methylcytosine immunoreactivity, Dnmt3 a protein levels and Dnmt enzyme activity increased preapoptotically. Inhibition of Dnmts with RG108 blocked completely the increase in 5methycytosine and the apoptosis of motor neurons in mice. In human amyotrophic lateral sclerosis (ALS), motor neurons showed changes in Dnmt1, Dnmt3a, and 5-methylcytosine similar to experimental models.

55. Everett CM, Wood NW. Trinucleotide repeats and neurodegenerative disease. Brain. 2004;127(Pt 11):2385-405.

56. Cleary JD, Pearson CE. The contribution of cis-elements to disease-associated repeat instability: clinical and experimental evidence. Cytogenet Genome Res. 2003;100(1-4):25-55.

57. Gatchel JR, Zoghbi HY. Diseases of unstable repeat expansion: mechanisms and common principles. Nat Rev Genet. 2005;6 (10):743-55.

58. Nichol K, Pearson CE. CpG methylation modifies the genetic stability of cloned repeat sequences. Genome Res. 2002;12(8):1246-56.

59. Ghoshal K, et al. 5-Aza-deoxycytidine induces selective degradation of DNA methyltransferase 1 by a proteasomal pathway that requires the KEN box, bromo-adjacent homology domain, and nuclear localization signal. Mol Cell Biol. 2005;25(11):4727-41.
60. Gorbunova V, et al. Genome-wide demethylation destabilizes CTG.CAG trinucleotide repeats in mammalian cells. Hum Mol Genet. 2004;13(23):2979-89.

61. Dion V, et al. Dnmt1 deficiency promotes CAG repeat expansion in the mouse germline. Hum Mol Genet. 2008;17(1):1306-17.

62. Coffee B, et al. Histone modifications depict an aberrantly heterochromatinized FMR1 gene in fragile x syndrome. Am J Hum Genet. 2002;71(1):923-32.

63. Pietrobono R, et al. Molecular dissection of the events leading to inactivation of the FMR1 gene. Hum Mol Genet. 2005;14(2):26777.

64. Taylor AK, et al. Tissue heterogeneity of the FMR1 mutation in a high-functioning male with fragile X syndrome. Am J Med Genet. 1999;84(3):233-9.

65. Tahiliani $M$, et al. Conversion of 5-methylcytosine to 5hydroxymethylcytosine in mammalian DNA by MLL partner TET1. Science. 2009;324(5929):930-5.

66. Kriaucionis S, Heintz N. The nuclear DNA base 5hydroxymethylcytosine is present in Purkinje neurons and the brain. Science. 2009;324(5929):929-30.

67. •• Guo JU, et al. Hydroxylation of 5-methylcytosine by TET1 promotes active DNA demethylation in the adult brain. Cell. 2011;145 (3):423-34. This paper showed that 5-methylcytosine ( $5 \mathrm{mC})$ hydroxylase TET1, by converting $5 \mathrm{mCs}$ to 5-hydroxymethylcytosines $(5 \mathrm{hm} C \mathrm{Cs})$, promotes DNA demethylation in mammalian cells through a process that requires the base excision repair pathway. Though expression of the 12 known human DNA glycosylases individually did not enhance removal of $5 \mathrm{hmCs}$ in mammalian cells, demethylation of both exogenously introduced and endogenous $5 \mathrm{hmCs}$ is promoted by the AID (activation-induced deaminase)/APOBEC (apolipoprotein B mRNA-editing enzyme complex) family of cytidine deaminases. Furthermore, Tet1 and Apobecl are involved in neuronal activity-induced, region-specific, active DNA demethylation and subsequent gene expression in the dentate gyrus of the adult mouse brain in vivo.

68. He YF, et al. Tet-mediated formation of 5-carboxylcytosine and its excision by TDG in mammalian DNA. Science. 2011;333 (6047):1303-7.

69. Iqbal K, et al. Reprogramming of the paternal genome upon fertilization involves genome-wide oxidation of 5-methylcytosine. Proc Natl Acad Sci U S A. 2011;108(1):3642-7.

70. Inoue A, Zhang Y. Replication-dependent loss of 5hydroxymethylcytosine in mouse preimplantation embryos. Science. 2011;334(6053):194. 